\title{
Development of Cable Accessories with SiR Insulation for 320kV HVDC Ca- bles
}

\author{
Ruoyu Xu ${ }^{1, *}$, Mingyu Zhou ${ }^{1}$, Zhengyi Han $^{1,2,3, * *}$, Yi Luo ${ }^{1}$, Haitian Wang ${ }^{1,3}$, Yuzhen Zhou ${ }^{1}$, and Tong Yang ${ }^{3}$ \\ ${ }^{1}$ Global Energy Interconnection Research Institute Europe, Kantstr. 162, 10623 Berlin, Germany \\ ${ }^{2}$ Politecnico di Torino, Corso Duca degli Abruzzi 24, 10129 Torino, Italy \\ ${ }^{3}$ State Key Laboratory of Advanced Power Transmission Technology (Global Energy Interconnection Research Institute Co., Ltd.,) \\ 102209 Beijing, China
}

\begin{abstract}
HVDC cable accessories with environment-friendly insulating material and flexible compatibility other than ethylene propylene diene monomer (EPDM) are demanded in the global market. In this paper, the authors present their newly developed silicone rubber ( $\mathrm{SiR}$ ) insulated cable joints and terminations as a potential answer to that call. Simulation models were built in computer programs. Three most venerable spots in the insulator were identified. SiR insulated accessories with optimised conductivity were produced and tested according to Cigre TB 496 and IEC 62895:2017. The test objects passed the type test with good success. It proved that with proper design, manufacturing and installation, $\mathrm{SiR}$ insulated cable accessories are able to provide good service in the power system networks.
\end{abstract}

\section{Introduction}

The growing needs for secure, clean and efficient energy [1] in Europe have driven extended utilisation of renewable sources. The cost of electricity from coal, gas, nuclear plants and petroleum keeps rising while the cost of renewable energy is clearly decreasing[2]. Wind energy, produced by off-shore wind farms, provides a practical solution. North Sea countries including Germany and UK are boosting their wind energy projects in the short-term and midterm [3]. Traditional HVAC cables in highly salted water environment see limitations in this scenario because the existence of large capacitance in the HVAC cables would require too much additional charging current which, in turn, significantly reduces the total volume of energy to be transmitted.

High-voltage direct current (HVDC) provides efficient non-synchronised bulk electric power transmissions with the economic benefits of reduced power loss, no reactive power, easy connection, and enhanced stability[2][4]. Free from reactive power loss, HVDC cables become viable solutions for submarine power transmission, offshore wind power integration, and harsh environment electrification[5].

Extruded power cables have obvious advantages over paper-lapped cables, such as a simpler production process, lower weight, simpler installation process due to easier jointing procedures, no maintenance, and environmentally friendly features[3]. The compatibility between Cross-linked polyethylene (XLPE) insulated power cable and ethylene propylene diene monomer (EPDM) rub-

\footnotetext{
* Corresponding author: ruoyu.xu@geiri.eu

** Corresponding author: zhengyi.han@geiri.eu
}

ber insulated cable joints as well as terminations (the most commonly used materials for power cables and cable accessories respectively) have been studied by many researchers.[6-9]

Despite the merits, which have been concluded in Ref.[10], that make EPDM viable for power cable, a non-negligible drawback factor is regrading environmental concerns. According to a commercial data sheet provided by Federal Morgnl, carbon black and other compositions of EPDM could be harmful for human body as well as for environment. Therefore, it is motivated to develop a new type of cable accessories with different insulating material.

In this paper, the authors present an alternative approach, namely silicone rubber ( $\mathrm{SiR})$, in a $320 \mathrm{kV}$ HVDC system. The prototype has passed the type test with good success.

\section{Modelling \& simulation}

Generally, the technical development for the SiR type cable accessories involves two major phases: Modelling \& Simulation and Type Test. In this section, the electrical field distribution along the SiR and XLPE interface will be studied.

\subsection{Material conductivity and effect}

When a power cable is operated at normal load conditions, a temperature gradient is formed because of the heat generated by the wire-core. Material conductivity and its relationship with electrical field and temperature can be mod- 
Table 1: Electrical conductivity fitting parameters of insulating materials

\begin{tabular}{lccc}
\hline Material & $A$ & $\psi$ & $B$ \\
\hline XLPE & $2.69 \times 10^{7}$ & 0.916 & $1.45 \times 10^{-7}$ \\
SiR & $3.14 \times 10^{4}$ & 0.674 & $1.41 \times 10^{-7}$ \\
\hline
\end{tabular}

Table 2: Character parameters for materials in cable accessories

\begin{tabular}{lccc}
\hline Material & $\sigma(\mathrm{S} / \mathrm{m})$ & $\epsilon$ & $\lambda\left(\mathrm{Wm}^{-1} \mathrm{~K}^{-1}\right)$ \\
\hline Copper & $5.8 \times 10^{7}$ & 1 & 398 \\
Semiconductive SiR & 100 & 1 & 100 \\
SiR for Accessories & $\sigma_{\text {SiR }}$ & 3 & 0.27 \\
XLPE & $\sigma_{X L P E}$ & 2.25 & 0.4 \\
\hline
\end{tabular}

elled with the following equation[11]:

$$
\sigma(T, E)=A_{1} \exp \left(-\frac{\psi q}{k T}\right) \frac{\sinh \left(B_{1} E\right)}{E}
$$

where $A$ and $B$ are constants, $\psi$ the thermal activation energy in $\mathrm{eV}, q$ the elementary charge, $T$ the temperature in $K, E$ the electrical field in $\mathrm{V} / \mathrm{m}$ and $k$ the Bolzmann constant.

The fitting parameters which have been adopted in our design are concluded in Table 1. At room temperature, the conductivity of XLPE is in the scale of $10^{-15} \mathrm{~S} / \mathrm{m}$ at $10 \mathrm{kV} / \mathrm{mm}$ electrical field. As for SiR, its conductivity is 30 times as much of that at the same condition. When the temperature rises to $80^{\circ} \mathrm{C}$, the conductivity of XLPE is $8 \times 10^{-13} \mathrm{~S} / \mathrm{m}$ whereas $\mathrm{SiR}$ is two times higher than XLPE.

Table 2 concludes the parameters of the electrical conductivity $\sigma$, relative permittivity $\epsilon$ and thermal conductivity $\lambda$ which have been utilised in our simulations.

\subsection{Fixed conductivity model}

The electrical field distribution under DC stress is mainly determined by the material conductivity. Specifically in our case, electrical field distribution in the HVDC cable joint/termination is under the influence of the conductivity of XLPE and SiR. A model with fixed conductivity values of $\sigma_{X L P E}$ and $\sigma_{S i R}$ was examined first for simplifications. $\sigma_{X L P E}=5 \times 10^{-14} \mathrm{~S} / \mathrm{m}$ was fixed in all cases while $\sigma_{S i R}$ was fixed at $5 \times 10^{-14} \mathrm{~S} / \mathrm{m}, 5 \times 10^{-13} \mathrm{~S} / \mathrm{m}$ and $5 \times 10^{-15} \mathrm{~S} / \mathrm{m}$ in case (a), (b) and (c), respectively. Fig. 1 shows the equal potential lines in the cable joint insulators at different conductivity ratios.

In comparison, the equal potential lines are more concentrated at the root of the stress cone in case (b) but at the semi-conductive electrode in case (c). On the other hand, the peak value of electrical field is found at inner side of XLPE/conductor interface in case (a) and (c) and the root of the stress cone in case (b). However, the concentrated equal potential lines at the semi-conductive electrode make the over electrical field in case (c) larger than case (a).
Therefore, it is believed that with similar conductivity values in XLPE and SiR, a better distributed electrical field can be achieved in the insulator of the cable joint.

\subsection{Dynamic conductivity model}

In reality, the conductivity values in XLPE and SiR vary with temperature and electrical field. When take the dynamically changed conductivity values into account, the electrical field distribution is more complicated.

Because the temperature at the vicinity of $\mathrm{XLPE} /$ conductor interface rises quickly, the conductivity becomes higher. On the contrary, the temperature at the vicinity of XLPE/SiR interface rises slowly which makes the conductivity lower. Therefore, the electrical field distribution reverses at this circumstance: the peak electrical field does not locate at the inner side of the XLPE/conductor interface but the outer side of the XLPE/SiR interface.

Fig. 4 shows the equal potential lines in the cable joint with conductivity optimised SiR. The data were recorded when the temperature of the ambient is $20^{\circ} \mathrm{C}$ and the cord of the conductor is $80^{\circ} \mathrm{C}$. The termination of the highvoltage electrode, the root to the stress cone and the outer side of the XLPE/SiR interface were identified the three most venerable spots based on our simulation.

\section{Tests}

\subsection{Tests arrangement}

Started from November 2016 and ended upon June 2017, six tests were organised in one of our manufactories' laboratory. The first five were failed in the middle of the testing

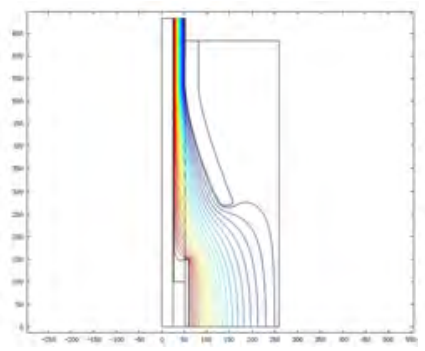

(a) $\sigma_{X L P E} / \sigma_{S i R}=1$

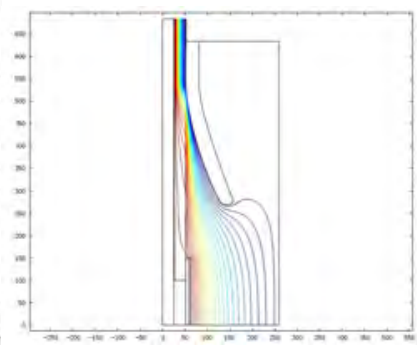

(b) $\sigma_{X L P E} / \sigma_{S i R}=10$

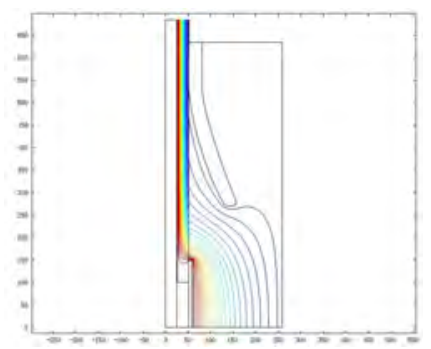

(c) $\sigma_{X L P E} / \sigma_{S i R}=0.1$

Fig. 1. Equal potential lines with different conductivity ratios 


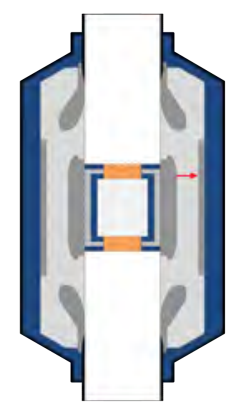

(a) Cable joint

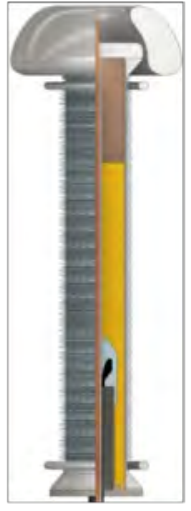

(b) Termination
Fig. 2. Cross section of the cable joint and termination in testing

processes and the last one survived all testing cycles with good success. Schematic cross section Figures of cable joints and terminations which have been used in the type test are shown in Fig. 2a and $2 b$.

Circuit layout and testing procedures were arranged according to the requirements by IEC 62895:2017 and instructions by Cigre TB496. Fig. 3a presents the testing circuit and $3 \mathrm{~b}$ is the actual setup at the testing site. Test objects produced by two manufactures were involved in

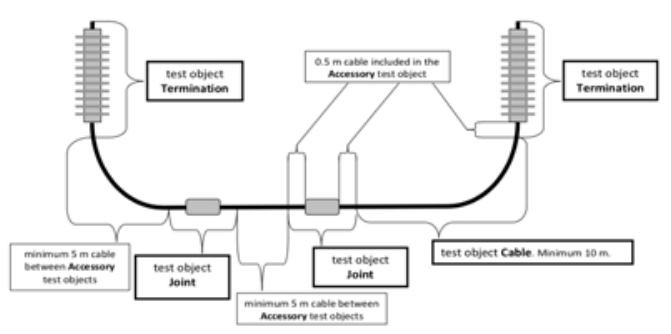

(a) Schematic circuit layout

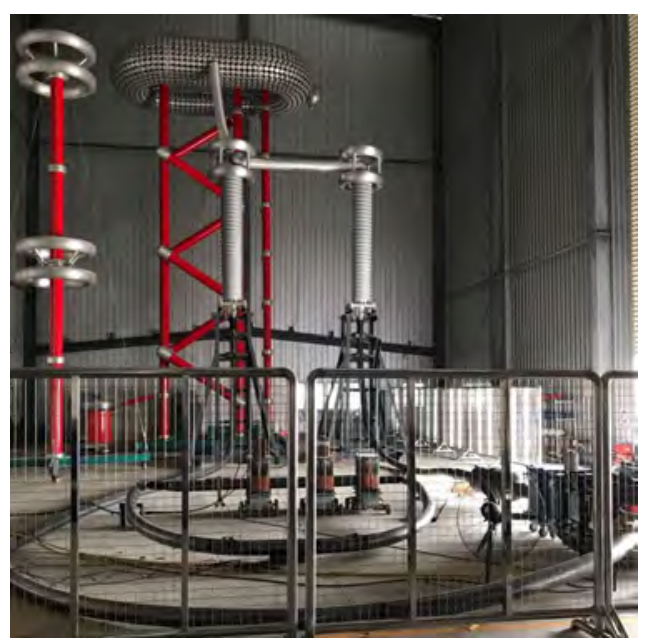

(b) Test circuit on site

Fig. 3. Test circuit
Table 3: Results of load cycle tests

\begin{tabular}{ccccccc}
\hline No. & Cable & Joint & \multicolumn{1}{c}{ Term. A } & Term. B & PD & Failure \\
\hline 1 & $\mathrm{X}$ & $\mathrm{X}$ & $\mathrm{Y}$ & $\mathrm{Y}$ & - & $1.5 \mathrm{~h}$ at the 6th cycle \\
2 & $\mathrm{X}$ & $\mathrm{Y}$ & $\mathrm{Y}$ & $\mathrm{Y}$ & $2.1 \mathrm{pC}$ & 3h at the 7th cycle \\
3 & $\mathrm{Y}$ & - & $\mathrm{Y}$ & $\mathrm{X}$ & $60 \mathrm{pC}$ & $1.5 \mathrm{~h}$ at the 1st cycle \\
4 & $\mathrm{Y}$ & - & $\mathrm{Y}$ & $\mathrm{X}$ & $2.4 \mathrm{pC}$ & $23 \mathrm{~h}$ at the 1st cycle \\
5 & $\mathrm{Y}$ & - & $\mathrm{Y}$ & $\mathrm{X}$ & $6.4 \mathrm{pC}$ & 4h at the 1st cycle \\
6 & $\mathrm{X}$ & $\mathrm{Y}$ & $\mathrm{Y}$ & $\mathrm{Y}$ & $1.9 \mathrm{pC}$ & No failure \\
\hline
\end{tabular}

our factory test procedures, marked as $\mathrm{X}$ and $\mathrm{Y}$ in this paper.

After "Non-electrical types tests" and "Mechanical preconditioning before electrical type test" have been done, test objects were subjected to electrical load cycle test. In our specific case, VSC testing route was followed. Therefore, with testing voltage $U_{T}=592 \mathrm{kV}$ stressed on the terminations, performances of all involved test objects were recorded cycle by cycle until failure occurs or the end of the last test cycle had been passed without failure.

\subsection{Tests results and failure analysis}

Table 3 concludes the overall results of electrical load cycle tests preformed in the laboratory. Red marked items in the table indicate failure was found on those objects whereas green marked items indicate no failure. The results provide that there is a clear link between detected partial discharge and possibility of total failure. Stronger partial discharge normally brings earlier failure. (In the first test, we did not measure PD)

In test one, insulator breakdown was found at the root of the deflector of the cable joint during the 6th cycle. Analysis indicates that the failure was mainly caused by imperfect installation. Fig. 5 clearly shows a gap between the curved body of the cable and the table. In test two, the testing cable was straightened under $85^{\circ} \mathrm{C}$ for 72 hours prior to the load test. PD measuring was added in this test. Breakdown was found at the high-voltage electrode during the 7 th cycle. It was identified that, again, imperfect

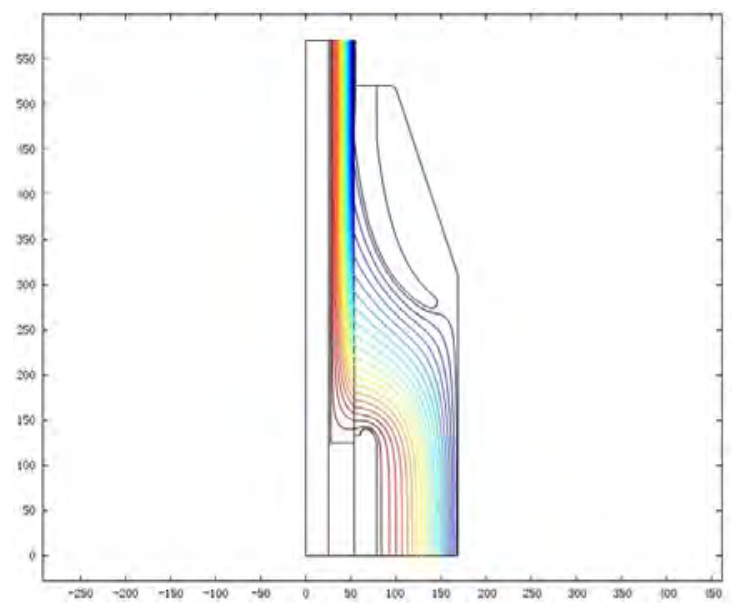

Fig. 4. Equal potential lines with optimised SiR conductivity 
installation caused the failure as metal screws were found in the joint. The metal screws brought extensive contact resistance at the joint which, therefore, overheated the insulator and caused the breakdown.

In test three, cable joints were removed to simplify the analysis. Breakdown was observed at the 1 st cycle on Termination A. Again, it was caused by imperfect installation: no fixing mechanism was installed at the rear end of the termination to fix the cable, and the deflector was not placed at a proper location. Significant pronounced PD signals as much as $60 \mathrm{pC}$ were detected in this test.

In test four, Termination A was replaced by a new one from the same manufacture. The breakdown was recorded at the 1st cycle on Termination B. As indicated in Fig. 7a and $7 \mathrm{~b}$, breakdown was initiated at the spot where is $1 \mathrm{~cm}$ below the root of the deflector. According to our simulation results, electrical field is supposed to be distributed smoothly in this area. It is believed that surface conditioning was not well performed on the semi-conductive shield. Besides, it was also found that the stress cone of the joint was misplaced in Termination B. Fig. 6 gives the electrical tree which has been found in the insulator.

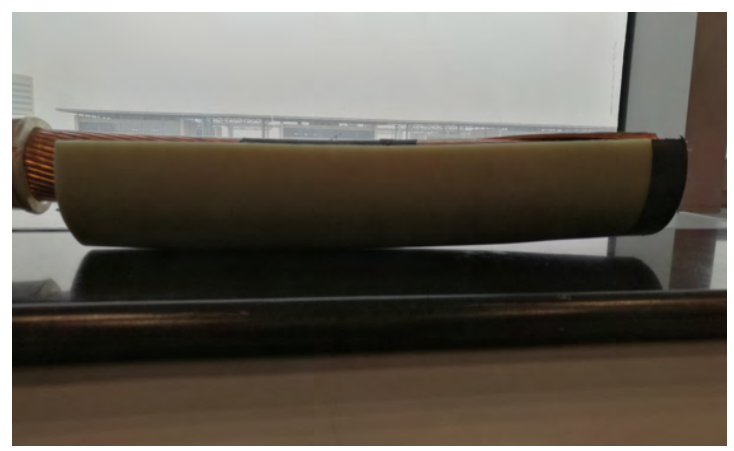

Fig. 5. Non-straight cable body

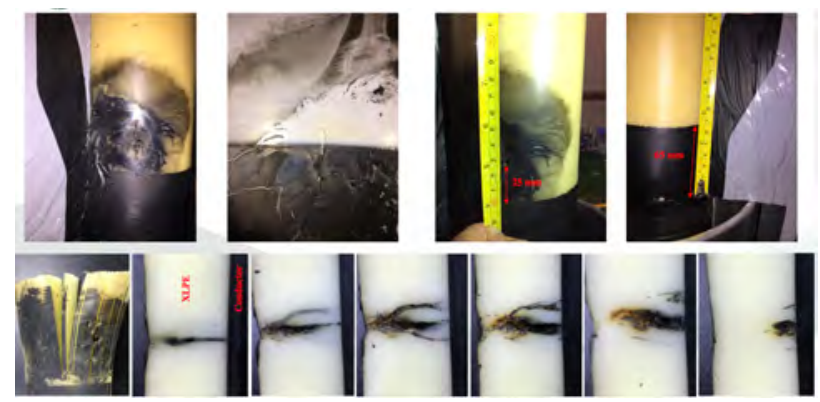

Fig. 6. Electrical tree in the insulator (test four)

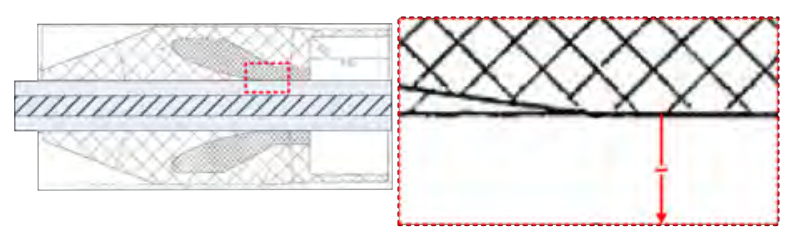

(a) Failure spot

(b) Zoom-in of the failure spot

Fig. 7. Failure analysis of test four

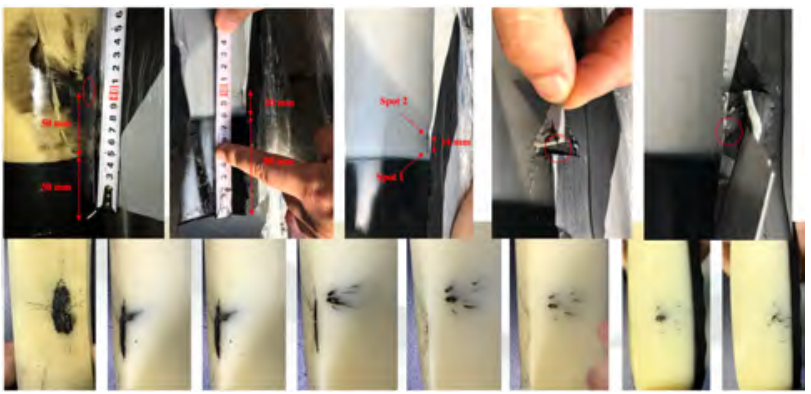

Fig. 8. Electrical tree in the insulator (test five)

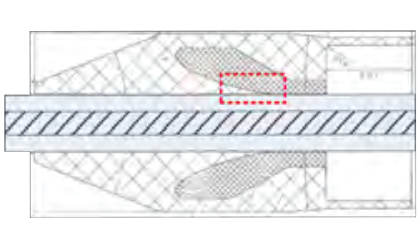

(a) Failure spot

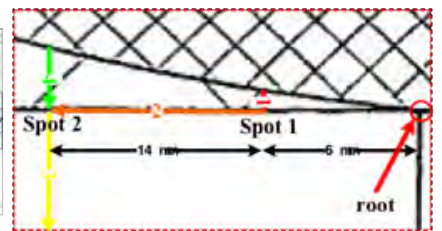

(b) Zoom-in of the failure spot
Fig. 9. Failure analysis of test five

In test five, the breakdown was still found at the 1 st cycle on Termination B. In this case, the initiating spot was located $6 \mathrm{~mm}$ above the root of the deflector (spot 1 ). Then it developed along with the interface to spot 2. Electrical tree was formed here. The maximum length of the tree was $14 \mathrm{~mm}$ and it reached spot 3 on the conductor. Protection relay triggered and the dielectric strength on the interface recovered. After that, the SiR insulator was broken down at spot 4. Important spots are highlighted in Fig. 9a and $9 \mathrm{~b}$. The reasons behind this symptom are the thickness of the cable shield was not evenly distributed and the interface pressure was over stressed.

In test six, no failures were found in cable joints or terminations.

\subsection{Summary}

With five failures and one success, it proved that our designs are able to provide qualified products. The major challenges lie with manufacturing process and installation process. Particularly, one has to keep in mind that:

- Power cables shall always be straightened with certain level of temperature before use.

- Metal screws shall be avoided at cable joints and terminations but metal fixing mechanism are needed for terminations.

- Surface conditioning is vitally important for the performance of the insulating.

- Thickness of the cable shield shall be maintained as evenly distributed as possible.

It is also worth noting that prior to the load test, a PD test provides an effective approach for early failure detection. 


\section{Conclusion}

Based on the R\&D experiences obtained from the aforementioned experiments, our HVDC SiR insulated cable accessories have passed two consecutive type tests in CNAS(China National Accreditation Service for Conformity Assessment) certification laboratory. In conclusion, our work has proven that the HVDC SiR insulated extruded cable joints and terminations are able to deliver similar insulating performance as what has been achieved in EPDM types. Yet, SiR insulated cable accessories are more friendly to the environment and compatible with cables produced by other manufacturers. Special concerns are required from the perspectives of manufacturing and installing processes.

This work was supported by the State Grid Corporation of China in its project entitled "Diagnostics of HVDC Cable Insulating Systems" (No. SGRI-ZL-71-16-036) and the National Key Research and Development Program of China "Key Technologies of Design and Manufacturing for $\pm 500 k V$ HVDC Cable Systems" (No. 2016YFB0900730) .

\section{References}

1. European Commission, Horizon 2020 work programme 2018-2020, 10. secure, clean and efficient energy (2018), http://ec.europa.eu/ research/participants/data/ref/h2020/wp/ 2018-2020/main/h2020-wp1820-energy_en. pdf

2. D. Fabiani, G.C. Montanari, C. Laurent, G. Teyssedre, P.H.F. Morshuis, R. Bodega, L.A. Dissado, A. Campus, U.H. Nilsson, IEEE Electrical Insulation Magazine 23, 11 (2007)
3. H. Ghorbani, M. Jeroense, C. Olsson, M. Saltzer, IEEE Transactions on Power Delivery 29, 414 (2014)

4. S. Delpino, D. Fabiani, G.C. Montanari, C. Laurent, G. Teyssedre, P.H.F. Morshuis, R. Bodega, L.A. Dissado, IEEE Electrical Insulation Magazine 24, 14 (2008)

5. H. Uehara, Z. Li, Q. Chen, G. Montanari, Y. Cao, Sensors and Materials 29, 1199 (2017)

6. S. Yi, Y. Wang, W. Li, J. Wu, Y. Yin, Research on the polarization relaxation current and equivalent circuit of double-layered XLPE/EPDM, in 2017 International Symposium on Electrical Insulating Materials (ISEIM) (2017), Vol. 1, pp. 327-330

7. W.C. Choi, S.K. Han, C.J. Lee, K.S. Park, S.O. Han, Breakdown characteristics of XLPE/EPDM on the treatment condition of the interfacial layer, in Proceedings of 5th International Conference on Properties and Applications of Dielectric Materials (1997), Vol. 1, pp. 345-348 vol.1

8. W.C. Choi, J.Y. Yoon, K.S. Park, S.O. Han, Electrical characteristics of the XLPE/EPDM double layered interface at the power cable joint, in Conference Record of the 1998 IEEE International Symposium on Electrical Insulation (Cat. No.98CH36239) (1998), Vol. 1, pp. 122-125 vol.1, ISBN 1089-084X

9. C. Stancu, P.V. Notingher, P. Notingher, M. Lungulescu, IEEE Transactions on Dielectrics and Electrical Insulation 23, 633 (2016)

10. D. Quaggia, Development of Joints $\mathcal{E}$ Terminations for HVDC Extruded Cables, in 2015 INMR World Congress: Insulators, Arresters, Bushings, Cable Accessories (2015), Vol. 1, pp. 228-234

11. A. Blythe, T. Blythe, D. Bloor, Electrical Properties of Polymers (Cambridge University Press, 2005), ISBN 9780521552196, https://books.google. de/books?id=NgVoPVNtjoAC 